\title{
ANALYSIS OF MECHANICAL FAULT DIAGNOSIS METHOD ACCORDING TO SIGNAL DEEP AUTOENCODER
}

\author{
Lisha Shang ${ }^{1 *}$ Yajuan Jia ${ }^{1}$, Junhui Liu ${ }^{1}$ \\ ${ }_{1} X i^{\prime} a n$ Traffic Engineering Institute, Xi'an, China. \\ Email: shanglisha09090@126.com
}

\begin{abstract}
This study aims to effectively carry out the health monitoring and fault diagnosis of mechanical equipment, predict and identify the faults in the operation of mechanical equipment in real time, and ensure the safe and stable operation of industrial system to avoid major catastrophic accidents. In this research, a mechanical fault diagnosis method based on the deep learning theory and signal depth automatic compression encoder is proposed, which is a combined fault diagnosis network with feature robustness and sparse enhancement. In this network, the depth sparse automatic encoder and depth compression automatic encoder are applied at the same time, and the Softmax classifier is combined to jointly complete the three stages of signal acquisition, feature extraction, and fault recognition and diagnosis, so as to realize the effective extraction of mechanical equipment fault features. The self-designed network in this research can simultaneously improve the robustness and sparsity of the extracted features, so as to enhance its expression ability, realize the effective diagnosis of mechanical faults under time-domain signals, and fully meet the needs of fault diagnosis during the operation of complex mechanical systems. The actual bearing experimental results proves that the enhanced joint fault diagnosis network with robustness and sparsity has a diagnostic accuracy of $98 \%$ under the condition of two hidden layers. Compared with the shallow machine learning method, it is shown that the mechanical fault diagnosis method based on signal depth automatic compression encoder can effectively realize the integration and intelligence of mechanical fault feature extraction and fault diagnosis. This method not only removes human intervention, but also has higher diagnostic accuracy. This study not only extends the research of deep autoencoder, but also provides a new idea and technical support for mechanical fault diagnosis.
\end{abstract}

Keywords: Deep Auto Encoder; Deep Learning; Machine Fault Diagnosis; Fault Feature Extraction.

\section{Introduction}

With the rapid development of science and technology and the upgrading of traditional industries, modern industrial systems have been developing towards the direction of large-scale complexity, and mechanical equipment has also grown in scale, sophisticated structures, diverse functions, and intelligent systems [1, 2]. Mechanical equipment plays a key role in the modern industrial system. If a certain component of a mechanical equipment fails, it will cause a series of chain reactions, which will make the whole industrial system unable to operate normally, cause huge losses to the national economy, and lead to the death of personnel in serious cases. With the rapid development of mechanical equipment, the health status monitoring and fault diagnosis of mechanical equipment have posed more severe challenges. It should establish a stable mechanical equipment health monitoring system, timely and accurately identify possible failures of machinery and equipment during operation, so that machinery and equipment can operate safely, thereby effectively reducing the probability of major catastrophic accidents.

Fault diagnosis technology is a key technology to improve the reliability and maintainability of mechanical equipment. It uses modern state monitoring methods and diagnosis theories to identify and diagnose the location, mechanism, and severity of possible faults, and determines corresponding maintenance measures and schemes according to the diagnosis results [3]. Relevant data show that by adopting fault diagnosis technology, the manufacturing industry can effectively improve the continuity of equipment operation, reduce the economic losses caused by the shutdown, and increase economic benefits. But at present, the failures of modern machinery and equipment have shown diversity, complexity, delay, hierarchy, and uncertainty. Traditional fault diagnosis methods obviously can't solve the problems in modern industrial systems.

Therefore, how to extract fault characteristics from massive data and identify mechanical 
equipment faults efficiently and accurately has become a hotspot and difficulty in the field of fault diagnosis [4, 5]. Faced with a series of technical problems in fault diagnosis methods of industrial systems, deep learning has shown unique advantages and potentials in feature extraction and pattern recognition, and the application of deep learning in fault diagnosis of complex mechanical equipment operating systems has mushroomed as the times require. Deep learning not only gets rid of the dependence on staff experience in the process of sample feature extraction, but also solves the problems existing in traditional methods such as gradient disappearance and local extremum, so as to meet the needs of fault diagnosis of modern mechanical equipment [6-8].

In the traditional fault diagnosis method for industrial system with complex structure, the feature extraction is difficult and the diagnosis effect is not ideal. In this research, based on the deep learning theory of signal deep autoencoder and combined with the Softmax classifier, the mechanical fault diagnosis is studied efficiently and accurately. To meet the needs of different mechanical equipment in practical application, a specific fault diagnosis network is designed. The effectiveness of the fault diagnosis network based on the signal deep autoencoder in mechanical fault diagnosis is proved by the actual bearing signal. It is hoped that the results of this study can provide certain technical support for the research of mechanical fault diagnosis methods in the later period.

\section{Literature Review}

Since the 1960s and 1970s, many foreign countries have successively carried out the research on fault diagnosis technology, and the mechanical fault diagnosis technology has developed rapidly $[9,10]$. Deutsch et al. (2017) used deep learning-based methods to predict the remaining useful life of rotating components in mechanical systems, tested, and validated the proposed method with the data collected from gear and bearing test benches, and compared it with existing prediction and health management system methods. The test results showed that the deep learning-based method had a good performance in predicting the residual service life of the rotating component [11]. Jang (2018) et al. proposed a fault detection technology based on machine learning and dual sensing to avoid sudden failure of mechanical structures under repeated loads, supplemented the traditional mechanical life management method, and determined the decision boundary through logistic regression. The results showed that the detection system had detected the fault before the crack appeared, and the maintenance time could be controlled [12]. The performance of traditional intelligent fault diagnosis methods depends on the feature extraction efficiency of fault signals. Hoang et al. (2019) proposed a bearing fault diagnosis method based on convolutional neural network depth structure. This method directly uses vibration signals as input data and is an automatic fault diagnosis system, which does not require any feature extraction technology and has high accuracy and robustness in noisy environment [13]. Buono (2017) et al. used ARMA model to analyse vibration signals. According to the analysis results, the monitoring and fault diagnosis can achieve real-time and high-precision fault identification and diagnosis of the thermodynamic pump in the running state [14]. At present, most of the researches on the fault detection of gear vibration are carried out under the condition of constant speed, but the researches based on the change of rotating speed are relatively few. Park (2019) et al. proposed a new method for detecting planetary gear faults under variable speed conditions, that is, the positive energy residual method. This method does not only need to assume small fluctuations in velocity, but also does not need angle information of gears. It is applicable to the study of gear fluctuations with large velocity, and the effectiveness of this method is proved by combining simulation and experiment [15].

At present, most researchers are committed to adopting deep learning to realize the qualitative diagnosis of fault types, but there are few studies on how to use deep learning to directly capture representative and effective features from the original time-domain signals. In this study, aiming at the defects of current mechanical fault diagnosis, deep learning method is used to carry out the research on mechanical fault diagnosis method based on signal deep autoencoder. In this method, multiple automatic encoders are stacked to build the deep autoencoders, and features are extracted automatically through the nonlinear transformation between different layers. In addition, Softmax classifier is used to realize automatic recognition and classification of fault features, so as to achieve integrated and intelligent fault feature extraction and fault recognition diagnosis and provide technical support for the research of deep autoencoders on mechanical fault diagnosis.

\section{Methodology}

\subsection{Construction of neural networks based on deep learning}

Deep learning is a new achievement of pattern recognition and machine learning, which belongs to unsupervised feature learning method and has a broad application prospect in equipment fault diagnosis. In this study, the established deep learning neural network model is shown in figure 1 . Its structure is similar to that of artificial neural network, except that deep learning neural network 
has multiple representations of hidden layers. Deep learning simulates the information processing mechanism of the human brain, trains the network structure, and learns and interprets input data. It has strong self-adaptability, automatically adjusts the characteristics of the input data, and also continuously updates the weight of the deep learning network, which further improves the ability to extract and learn fault features. In deep learning neural network, each layer learns deeper features based on the output of the previous layer. As the number of neural network layers' increases, each layer reconstructs the features from the previous layer, and the recognized features become more complex. The deep learning neural network framework combines feature extraction and classifier, which does not depend on the experience of the staff, reduces the computation, and improves the work efficiency. According to the input sample data and feature representation, learning methods can be detected and classified by themselves, realizing intelligent operation, which is very convenient.

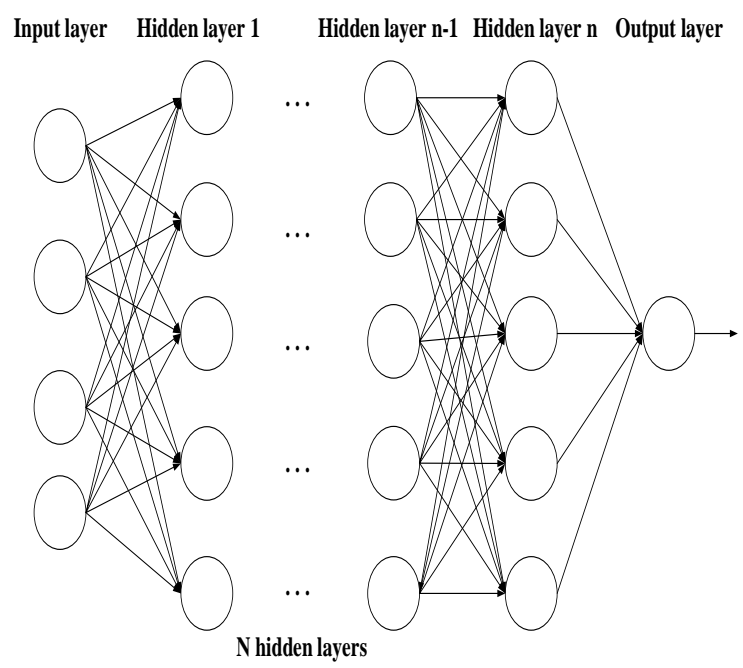

Figure 1: Schematic diagram of deep learning network structure

\subsection{Self-designed automatic encoder}

The automatic encoder is an unsupervised selflearning mode with three fully connected neural network structures, including the input layer, the hidden layer, and the output layer. The encoder can map the input data from the high-dimensional space into the code in the low-dimensional space, and the decoder reconstructs the output data in the feature layer into the original input data, and obtains the effective features of the input data from the hidden layer through the encoding process and decoding process [16]. In this research, the constructed automatic encoder structure is shown in figure 2 .
The input layer and the hidden layer together constitute the coding network $\mathrm{h}=\mathrm{f}(\mathrm{x})$, the hidden layer and the output layer constitute the decoding network $y=f(h)$. Among them, $x=x_{1}, x_{2}, \ldots, x_{n}$ represents the input value, $h=h_{1}, h_{2}, \ldots, h_{n}$ stands for hidden value, $\mathrm{z}=\mathrm{Z}_{1}, \mathrm{Z}_{2}, \ldots, \mathrm{Z}_{\mathrm{n}}$ is the output value. The input data of the auto-encoder is the same as the output target, that is, the vector set $\left\{\mathrm{x}^{\mathrm{m}}\right\}$ is encoded into the lowdimensional $\left\{\mathrm{h}^{\mathrm{m}}\right\}$. $\left\{\mathrm{h}^{\mathrm{m}}\right\}$ is converted to $\left\{\mathrm{z}^{\mathrm{m}}\right\}$ after inverse encoding. If the input result $\left\{\mathrm{x}^{\mathrm{m}}\right\}$ is consistent with the output result $\left\{\mathrm{z}^{\mathrm{m}}\right\}$ in this process, the encoding vector $\mathrm{h}^{\mathrm{m}}$ is considered to reconstruct the input data $\mathrm{x}^{\mathrm{m}}$ well. In the above process, the output of the automatic encoder is shown as follows.

$$
h^{m}=f\left(\mathrm{~W}^{(1)} x^{m}+b^{(1)}\right)
$$

The following equation can be obtained by the reverse coding of feature extraction result $\mathrm{h}^{\mathrm{m}}$.

$$
z^{m}=g\left(\mathrm{~W}^{(2)} h^{m}+b^{(2)}\right)
$$

Where, $f$ and $g$ are the activation functions of the encoding and decoding networks, w (1) and w (2) are the connection weights of the encoding and decoding networks, and $b^{(1)}$ and $b^{(2)}$ are the biasing of the encoding and decoding networks.

The reconstruction error between the input $x^{m}$ and the reconstructed $\mathrm{z}^{\mathrm{m}}$ is the cost function of a single sample and can be expressed as equation 3 .

$$
L\left(x^{m}, z^{m}\right)=\frac{1}{2} \mathrm{P} x^{m}-z^{m} \mathrm{P}_{2}^{2}
$$

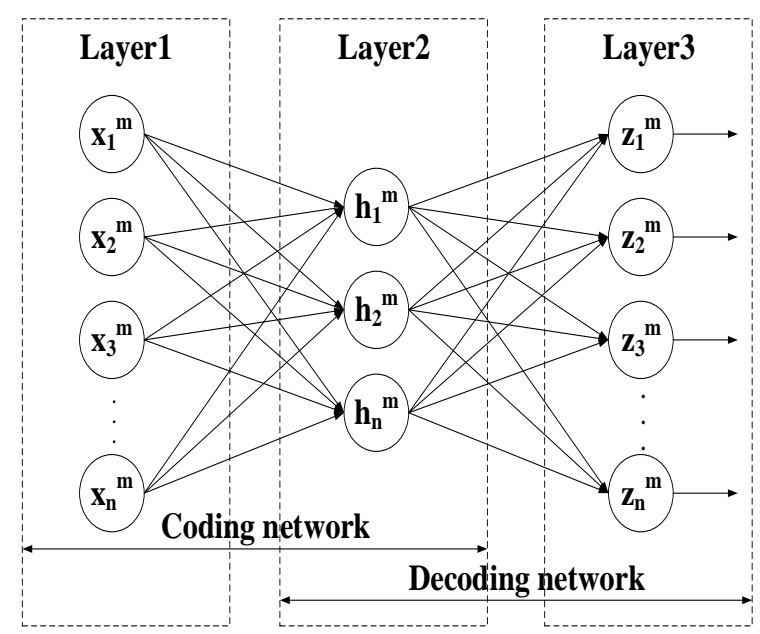

Figure 2: Schematic diagram of the structure of automatic encoder

During the operation of mechanical equipment, because the environment is noisy and the processes are complex and varied, the sample data obtained is susceptible to noise interference. Only relevant information of $\mathrm{x}^{\mathrm{m}}$ is retained, so the automatic encoder can't obtain the most effective feature representation. Therefore, in this research, by adding certain constraints to the automatic encoder, the automatic encoder can enhance the robustness of feature expression by reconstructing sample data containing noise. The specific de-noising process is 
shown in figure 3. When the sample data $\mathrm{x}^{\mathrm{m}}$ is input, a noise with a distribution form of $\mathrm{N}_{\mathrm{D}}$ is added to convert it to $\mathrm{x}^{\mathrm{m}}$.

Then, through the coding network, $\mathrm{x}^{\mathrm{m}}$ is encoded as the coding vector $\mathrm{h}^{\mathrm{m}}$. After the reconstruction through $\mathrm{h}^{\mathrm{m}}, \mathrm{z}^{\mathrm{m}}$ is output, the reconstruction error of $\mathrm{z}^{\mathrm{m}}$ is $\mathrm{R}(\mathrm{x}, \mathrm{y})$, and $\mathrm{x}^{\mathrm{m}}$ is minimized.

The stochastic gradient descent algorithm is used to optimize the cost function step by step to maximize the reconstruction of the original input data $\mathrm{x}^{\mathrm{m}}$.

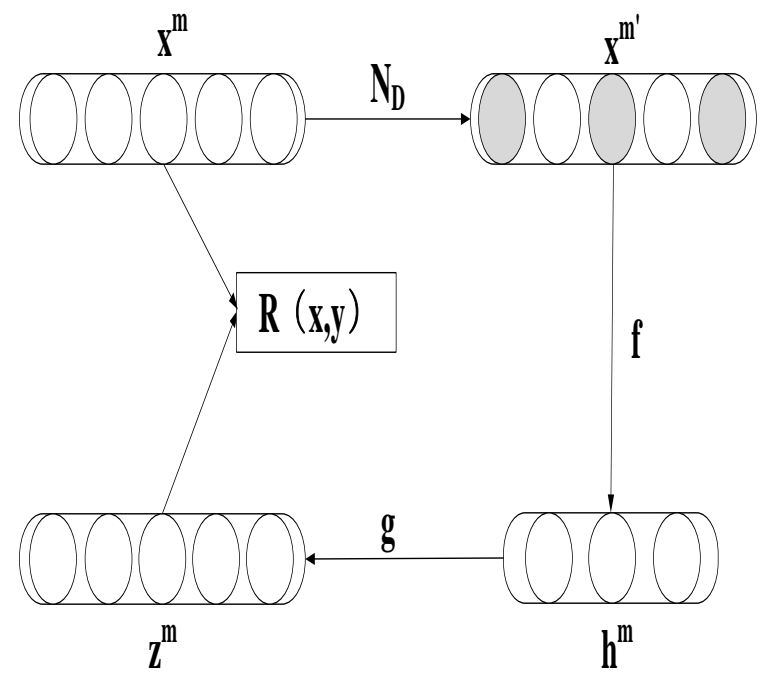

Figure 3: Flow chart of denoising principle of automatic encoder

\subsection{Frame of deep autoencoder}

The deep autoencoder is composed of multiple automatic encoders, which is mainly used for dimensionality reduction of high-dimensional data. According to the types of automatic encoders, deep autoencoders of various forms can be constructed, including sparse automatic encoders, noise reduction automatic encoders, and compression automatic encoders, etc. [17].

In this research, the network structure of deep autoencoder is divided into two parts, namely the encoding part and the decoding part. Its frame diagram is shown in figure 4.

The change from the input layer to the hidden layer is called coding.

This process is completed by the Encoder, which converts the data input from the higher dimensional space to the lower dimensional feature space.

The coding information obtained after coding is stored in the hidden layer.

The decoding process reconstructs the input data from the feature space through the Decoder, in other words, the decoding process is equivalent to the inverse process of encoding. After the original input data information is sent to the Encoder, a new Code is obtained after coding, which is another representation of the input.
Therefore, the parameters of Encoder and Decoder are constantly adjusted to minimize refactoring errors.

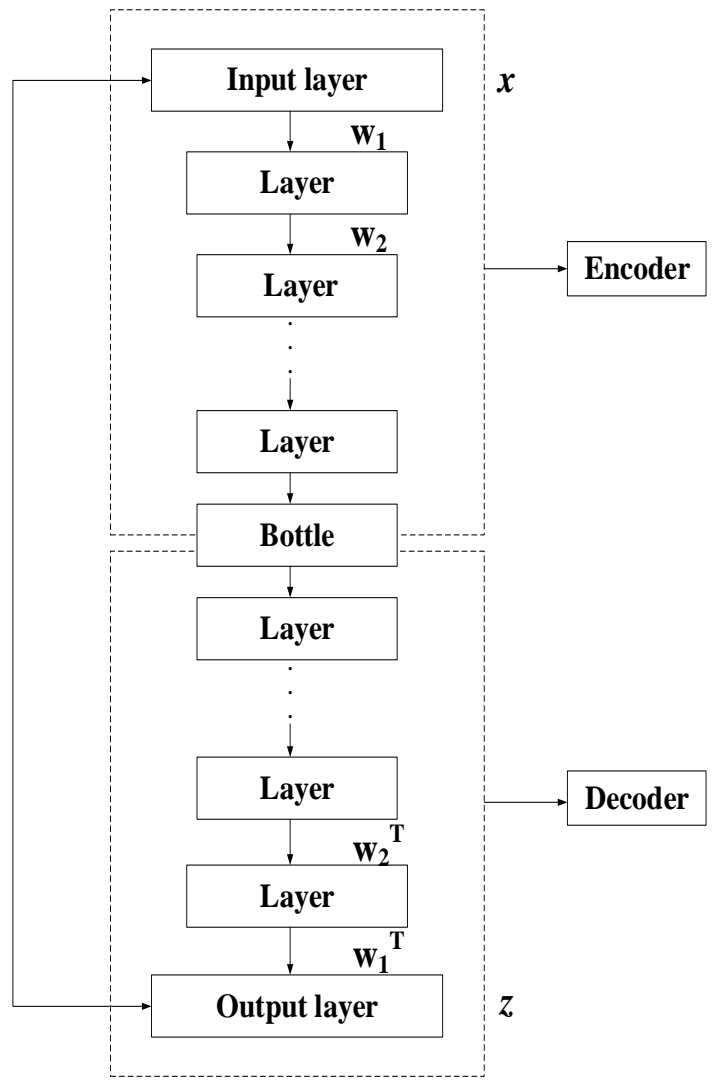

Figure 4: Frame diagram of the structure of the deep autoencoder

\subsection{Expression of Softmax classifier model}

To make efficient use of the extracted features in the process of fault diagnosis, the Softmax classifier is used to classify them in this research.

The logistic regression model is extended by the Softmax classifier. Compared with the logistic regression, which can only deal with nonlinear binary classification problems, the Softmax classifier can complete multi-classification problems.

Moreover, each classification strictly abides by the principle of mutual exclusion.

A sample can't occupy two classification categories at the same time, which can well solve the problem of multiple classification and give the probability of each category [18].

The structure of the Softmax classifier is shown in Figure 5, and its model is shown as follows.

$$
p_{\mathrm{w}(i)}(y=i \mid x)=\frac{1}{1+\exp \left(-W^{(i)^{T}} x\right)}
$$

Where, $p_{w(i)}(i=0,1)$ is the activation function with $\mathrm{w}(\mathrm{i})$ as the variable, $w(\mathrm{i})$ is acquired through learning of training set, and $x_{n}$ is the characteristic parameter acquired after deep neural network learning. 


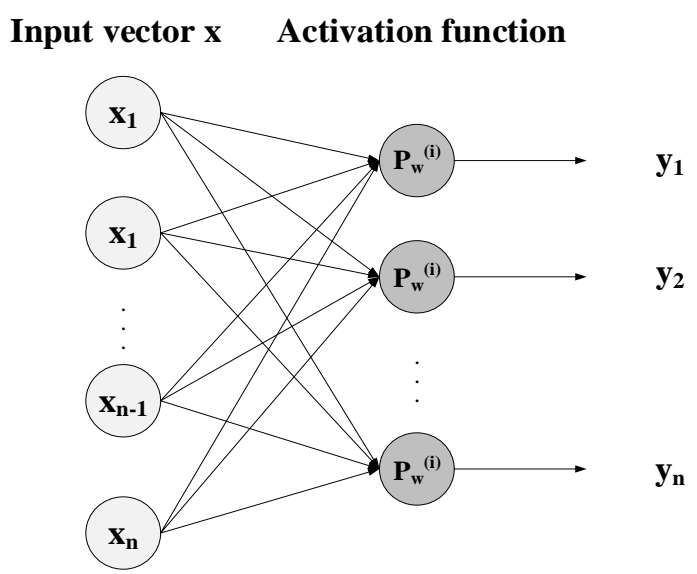

Figure 5: Structure of Softmax

\subsection{Adaptive fault diagnosis network based on signal deep autoencoder}

Whether feature extraction is effective or not is the key of mechanical fault diagnosis system. Sparsity is one of the key factors to measure fault characteristics, and the excessive redundancy features lack representativeness, which makes the identification and classification have strong interference.

Robustness is another key factor to measure features.

In practical fault diagnosis, the collected timedomain signals are usually interfered by noise, which reduces the quality of extracted features and makes diagnosis more difficult.

In addition, the convergence speed of diagnosis network also seriously affects the efficiency of fault diagnosis. The deep compression automatic encoder can automatically extract the intrinsic features of fault and effectively enhance its robustness, thus reducing the interference of noise.

To solve the perplexity of adopting time domain signals directly to realize mechanical fault diagnosis in practical applications, it proposes an enhanced joint fault diagnosis network of feature robustness and sparse by combining deep sparse automatic encoder and deep compression automatic encoder in this research.

The specific fault diagnosis network principle is shown in figure 6 .

Figure 7 shows the structure of the diagnostic network. Firstly, the compression automatic encoder is used to improve the robustness of features and reduce the interference of noise to time-domain signals. Then, the sparse automatic encoder is used to enhance the sparsity of features.

The robustness and sparsity of the features are enhanced while the features are extracted automatically, thus making the automatically extracted fault features more expressive.

Finally, combined with the Softmax classifier, the diagnosis of various mechanical fault types under the time domain signal with noise is effectively realized.

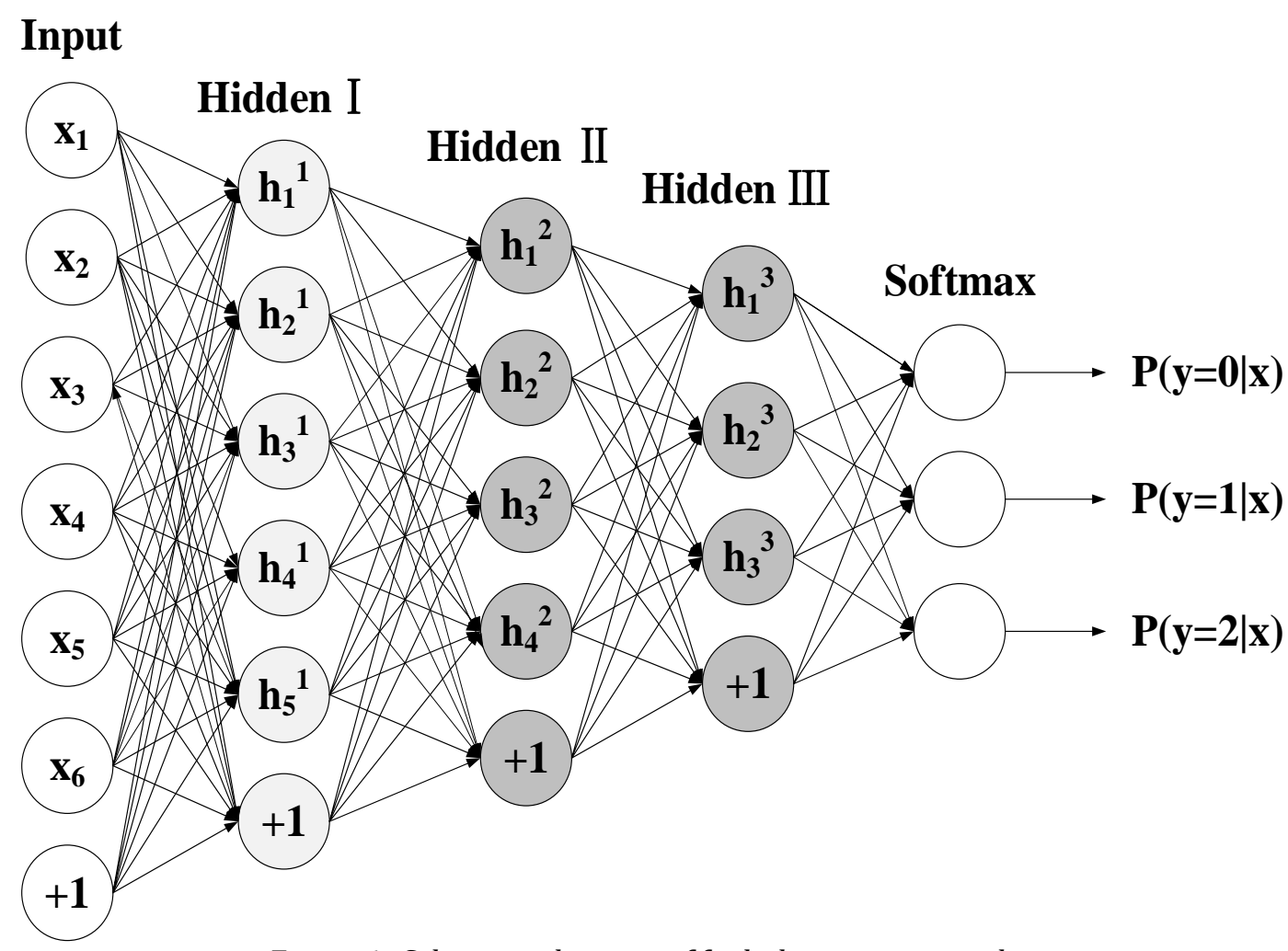

Figure 6: Schematic diagram of fault diagnosis network 


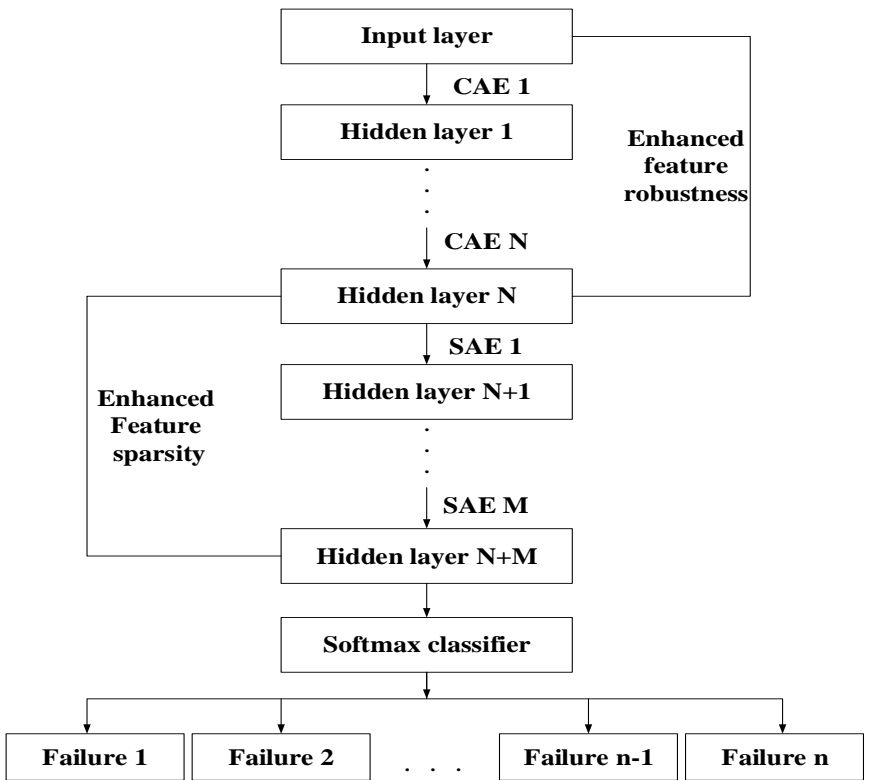

Figure 7: Structure of fault diagnosis network based on signal deep autoencoder

\subsection{The training process of fault diagnosis network based on signal deep autoencoder}

The training process of deep autoencoder is to obtain the best network parameters by continuously reducing the cost function and updating parameters, so as to achieve automatic learning of original input effective information and feature extraction, as shown in figure 8.

First, weight and bias are randomly initialized.

Then, the back propagation algorithm is used to carry out forward propagation, and the activation values of the hidden layer and the output layer are calculated respectively. Next, the process of back propagation is carried out.
The error is transmitted back to the input layer by the cost function and distributed to each layer. After calculating the gradient of each layer's parameters, the parameters are updated with the gradient descent method. The updated parameters are propagated forward and back, and the cost function value is reduced continuously until the maximum number of iterations is obtained.

The resulting weights and offsets are retained and the training process for the depth auto-encoder is completed.

Finally, the activation value of hidden layer is calculated, which can be used as fault characteristics automatically extracted by unsupervised learning in the training process of deep autoencoder.

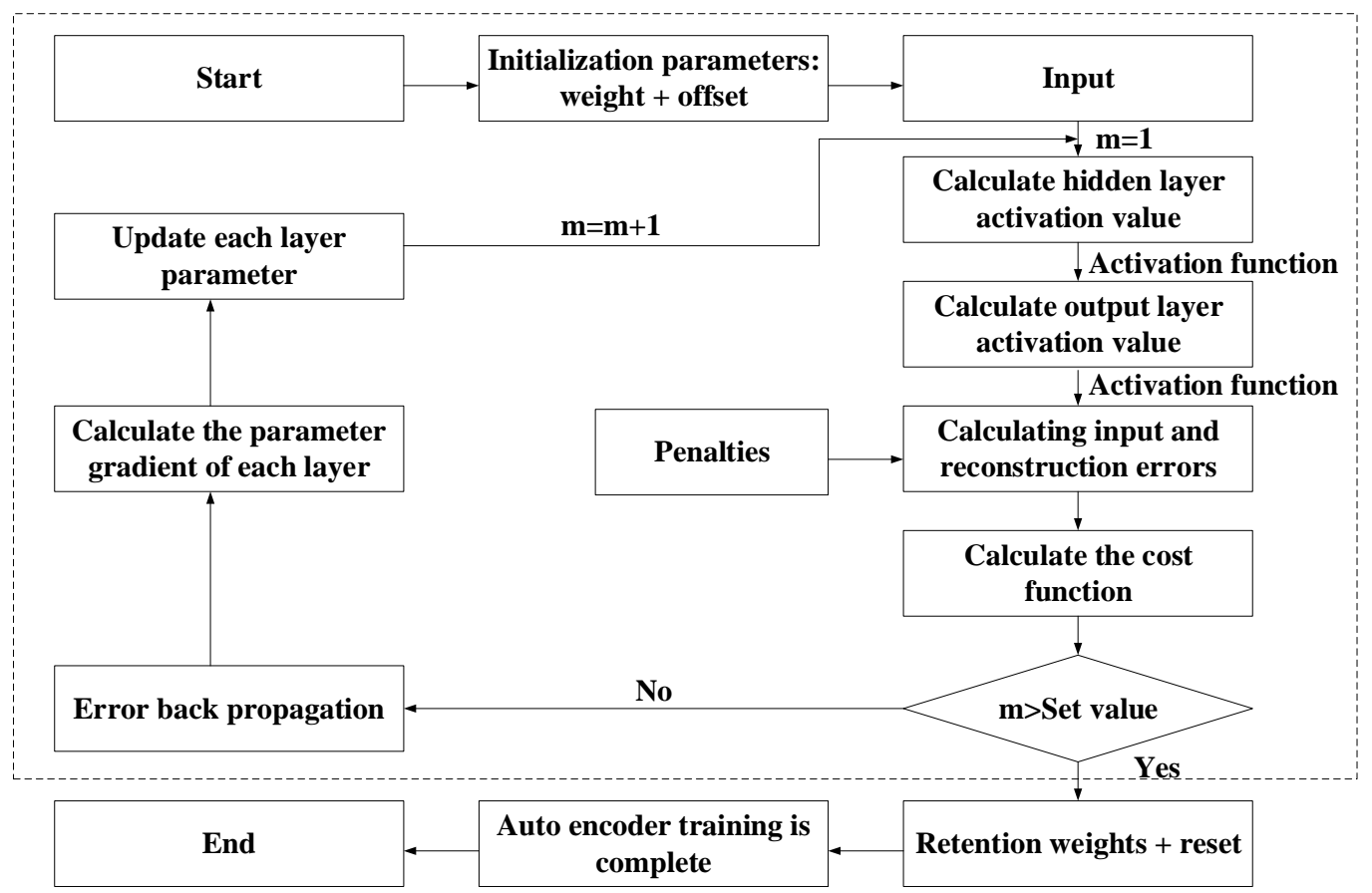

Figure 8: Training flow chart of fault diagnosis based on signal deep autoencoder 


\section{Results}

\subsection{Application of machine fault diagnosis model based on signal deep autoencoder}

The application process of fault diagnosis network based on signal deep autoencoder is shown in figure 9. The initial input of the network is the original time-domain signal. After signal preprocessing, it is divided into training samples and test samples. The training samples are pre-trained without supervision and fine-tuned with supervision through the established feature enhanced joint fault diagnosis network. After the diagnostic network is trained, it is used to identify and diagnose various fault types of test samples.

By comparing with the ideal fault type, the effectiveness of the actual diagnosis operation of the diagnostic network is verified.

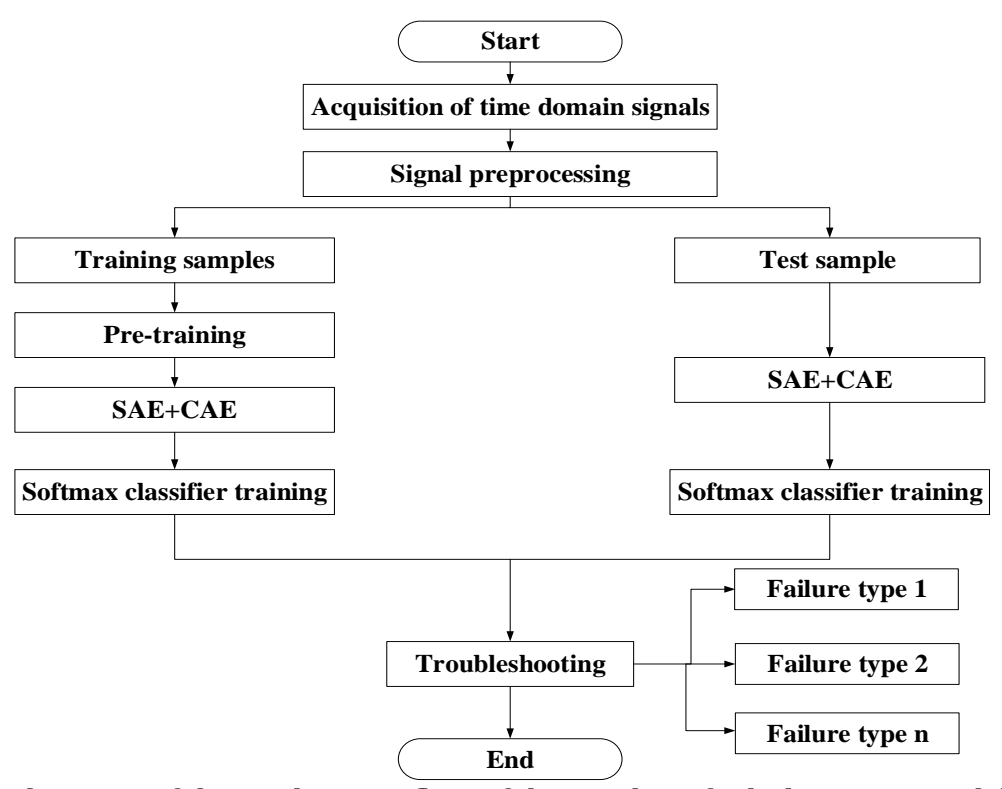

Figure 9: Schematic diagram of the application flow of the machine fault diagnosis model based on the signal automatic encoder

\subsection{Experimental verification results of bearing fault diagnosis}

Figure 10 is a histogram of the proportion of faults of each key component in the mechanical equipment. It can be concluded from the figure that the proportion of rolling bearing, rotor/stator, gear, engine, and other faults is $43 \%, 26 \%, 19 \%, 6 \%$, and $6 \%$, respectively. Bearing is an important component in mechanical system, and the proportion of faults is the highest. In the operation of the machinery, if there is a failure, it will directly lead to major economic losses and even casualties. Due to the severe and complicated working environment of the rolling bearing, the fault diagnosis effect is poor.

Therefore, an effective bearing fault diagnosis method is of great significance to the normal operation of mechanical equipment.

To verify the effectiveness of the self-designed fault diagnosis network based on the automatic encoder of signal depth, in this research, the bearing fault simulation experiment table prepared by the laboratory is used to collect bearing signals under different health conditions.

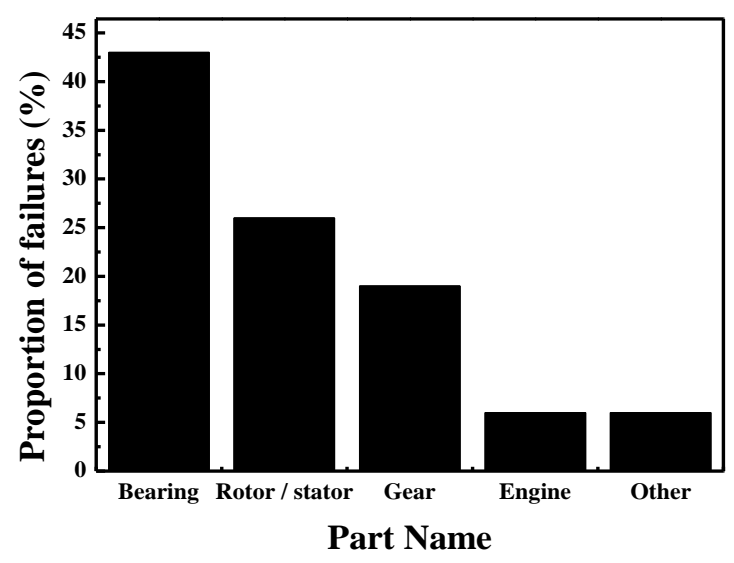

Figure 10: Proportion of failures of various components in mechanical equipment

The experiment table consists of motor, bolt-nut loading system, normal bearing, test bearing, and acceleration sensor. During the test, the acceleration sensor is placed on the test bearing seat and fixed in front of the test bearing. The NI pxle-4496 data acquisition system is adopted to collect the bearing vibration signals.

The test bearing type used in the experiment is FAG $6005-2 \mathrm{RS}$, which is a fault of $0.5 \mathrm{~mm}$ artificially 
cut on the inner ring, outer ring, and rolling body of the bearing by wire cutting.

The motor speed is 960rpm, the sampling frequency is set as $10000 \mathrm{~Hz}$, and the total number of sampling points is 1500 .

The 4 kinds of data collected are inner ring fault of bearing, outer ring fault of bearing, rolling body fault, and normal state, whose labels are defined as 01, 02, 03, and 04. 450 samples are collected under each healthy state, of which 300 are randomly selected as training samples and the rest as test samples. Bearing data set information is shown in Table 1.

In the fault diagnosis network based on the automatic encoder of signal depth, the compression penalty coefficient is set as 0.0001 , and the sparse parameter is 0.1 .

The fault diagnosis accuracy is shown in Table 2 .

Table 1. Bearing data set information

\begin{tabular}{|c|c|c|c|}
\hline Bearing state & Number of training samples & Number of test samples & Custom label \\
\hline Inner ring fault & 300 & 150 & 01 \\
\hline Outer ring fault & 300 & 150 & 02 \\
\hline Rolling body fault & 300 & 150 & 03 \\
\hline Normal status & 300 & 150 & 04 \\
\hline
\end{tabular}

Table 2. Bearing fault diagnosis accuracy based on signal automatic encoder under different hidden layers

\begin{tabular}{|c|c|c|c|c|}
\hline Diagnosis type & Two hidden layers & $\begin{array}{c}\text { Three hidden } \\
\text { layers }\end{array}$ & Four hidden layers & $\begin{array}{c}\text { Five hidden } \\
\text { layers }\end{array}$ \\
\hline Diagnosis accuracy (\%) & 98 & 92 & 87 & 81 \\
\hline
\end{tabular}

\section{Discussion}

According to the bearing failure experiment verification, when the number of hidden layers of the fault diagnosis network based on the signal depth autoencoder is the same, the highest diagnostic accuracy of time-domain signals obtained by a compression automatic encoder and then a sparse automatic encoder is higher than that obtained by a sparse automatic encoder and then a compression automatic encoder.

It is shown that in the diagnosis process, the compression automatic encoder is first used to enhance the robustness of fault characteristics, and then the sparse automatic encoder is used to improve the sparsity of fault characteristics, which is more conducive to the processing of time-domain signals under the interference of noise, so as to effectively identify and diagnose mechanical faults.

When the number of hidden layers is between 2 and 5 , the accuracy of mechanical fault diagnosis is more than $80 \%$, which fully verifies that this combined fault diagnosis network can directly extract fault features with strong expression ability from the original signal, get rid of human factors, and realize mechanical fault diagnosis in the time domain signal. At the same time, it is found that the diagnosis accuracy decreases with the increase of hidden layers. When the number of hidden layers is 2 , the combined fault diagnosis network can obtain the highest diagnosis accuracy of $98 \%$.

Compared with other mechanical fault diagnosis methods, the fault diagnosis network based on signal deep autoencoder proposed in this research integrates the advantages of sparse automatic encoder and compression automatic encoder. It makes the extracted features more representative; it is resistant to changes in input features, and has a stronger expression ability. It belongs to a special type of enhanced joint fault diagnosis network with sparsity and robustness.

In the process of diagnosis, the original time domain signal is directly processed, so that the size of mechanical fault can be quantified for diagnosis, and the identification and diagnosis of mechanical fault with high accuracy can be realized, which is particularly convenient.

\section{Conclusions}

To realize real-time and effective diagnosis of mechanical faults, a fault diagnosis method based on signal depth automatic compression encoder is proposed in this research, that is, the enhanced joint fault diagnosis network with the feature robustness and sparsity. The network combines the depth sparse automatic encoder with the depth compression automatic encoder. First, the deep learning method is adopted to maximize the mining of hidden features in data, give full play to the expressive force of samples, and realize the effective extraction of features. Then, the fault diagnosis model is trained by combining pre-training with finetuning.

Finally, the fault results are output by combining with the Softmax classifier. The bearing experiments show that the diagnosis accuracy of the enhanced joint fault diagnosis network with robustness and sparsity is more than $80 \%$, which can meet the requirements of fault diagnosis in the operation of complex mechanical industry systems, and the diagnosis accuracy is as high as 98\% under two hidden layers. Compared with the traditional mechanical fault diagnosis method, the mechanical 
fault diagnosis method based on the signal deep autoencoder and deep learning theory can autonomously learn the inherent hidden features of the data from the time-domain vibration signals of mechanical equipment, which makes the mechanical fault diagnosis efficient, intelligent, and accurate.

It gets rid of the reliance on diagnostic knowledge and diagnostic experience in traditional fault diagnosis technology, achieves adaptive extraction of fault characteristics of mechanical equipment and intelligent judgment of health status in a big data environment, and reduces the factors of manual participation.

In the construction process of deep autoencoder, many parameters have not formed a complete theoretical support so far, which are determined based on previous experience and a large number of experiments, with strong subjectivity and limited time, so many aspects still need to be further optimized in this study. To sum up, in this research, the signal-based deep automatic encoder is applied to the fault diagnosis of mechanical equipment, and the integrated and intelligent fault feature extraction and fault diagnosis of mechanical equipment are realized, which lays a foundation for the fault diagnosis research of mechanical equipment.

\section{References}

[1] Darong H., Lanyan K., Bo M, et al. (2018) A new incipient fault diagnosis method combining improved RLS and LMD algorithm for rolling bearings with strong background noise. IEEE Access, 6, 26001-26010.

[2] Zhang W., Lu S., Ding X. (2019) Recent development on innovation design of reconfigurable mechanisms in China. Frontiers of Mechanical Engineering, 14(1), 15-20.

[3] Sohaib M., Kim J. M. (2017) A Robust Deep Learning Based Fault Diagnosis of Rotary Machine Bearings. Advanced Science Letters, 23(12), 12797-12801.

[4] Kompella K. C. D., Rao M. V., Rao R. S, et al. (2017) Bearing fault diagnosis in 3 phase induction machine using current spectral subtraction with different wavelet transform techniques. Journal of Electrical Systems, 13(1), 143-159.

[5] Rajamani R., Rajappa M., Arunachalam K, et al. (2017) Interturn short diagnosis in small transformers through impulse injection: on-line on-load self-impedance transfer function approach. Iet Science Measurement \& Technology, 11(8), 961-966.

[6] Wurfl T., Hoffmann M., Christlein V, et al. (2018) Deep Learning Computed Tomography: Learning Projection-Domain Weights from Image Domain in Limited Angle Problems. IEEE Transactions on Medical Imaging, 37(6), 1454-1463.
[7] Shao S., McAleer S., Yan R, et al. (2018) Highly Accurate Machine Fault Diagnosis Using Deep Transfer Learning. IEEE Transactions on Industrial Informatics, 15(4), 2446-2455.

[8] Chellamuthu S., Sekaran E. C. (2019) Fault detection in electrical equipment's images by using optimal features with deep learning classifier. Multimedia Tools and Applications, 19, 27333-27350.

[9] Ahour J. N., Seyedtabaii S., Gharehpetian G. B. (2017) Determination and localisation of turn-toturn fault in transformer winding using frequency response analysis. IET Science, Measurement \& Technology, 12(3), 291-300.

[10] Glowacz A. (2019) Fault diagnosis of singlephase induction motor based on acoustic signals. Mechanical Systems and Signal Processing, 117, 65-80.

[11] Deutsch J., He D. (2017) Using deep learningbased approach to predict remaining useful life of rotating components. IEEE Transactions on Systems, Man, and Cybernetics: Systems, 48(1), 11-20.

[12] Jang D. W., Lee S., Park J. W, et al. (2018) Failure detection technique under random fatigue loading by machine learning and dual sensing on symmetric structure. International Journal of Fatigue, 114, 57-64.

[13] Hoang D. T., Kang H. J. (2019) Rolling element bearing fault diagnosis using convolutional neural network and vibration image. Cognitive Systems Research, 53, 42-50.

[14] Buono D., Siano D., Frosina E, et al. (2017) Gerotor pump cavitation monitoring and fault diagnosis using vibration analysis through the employment of auto-regressive-moving-average technique. Simulation Modelling Practice and Theory, 71, 61-82.

[15] Park J., Hamadache M., Ha J. M, et al. (2019) A positive energy residual (PER) based planetary gear fault detection method under variable speed conditions. Mechanical Systems and Signal Processing, 117, 347-360.

[16] Eravci B., Ferhatosmanoglu H. (2018) Diverse Relevance Feedback for Time Series with Autoencoder Based Summarizations. IEEE Transactions on Knowledge and Data Engineering, 30(12), 2298-2311.

[17] Chaitanya C. R. A., Kaplanyan A. S., Schied C, et al. (2017) Interactive reconstruction of Monte Carlo image sequences using a recurrent denoising autoencoder. ACM Transactions on Graphics, 36(4), 98.

[18] Khanna B., Moses S., Nirmala M. (2018) SoftMax based User Attitude Detection Algorithm for Sentimental Analysis. Procedia Computer Science, 125, 313-320. 